\title{
Monophonie, hétérophonie et poly(?)phonie dans le jeu du violon traditionnel en Pologne
}

Mono-, hetero- (and poly-?)phony in traditional fiddling in Poland

\section{Ewa Dahlig}

Traducteur : Isabelle Schulte-Tenckhoff

\section{(2) OpenEdition \\ Journals}

Édition électronique

URL : http://journals.openedition.org/ethnomusicologie/1386

ISSN : 2235-7688

Éditeur

ADEM - Ateliers d'ethnomusicologie

Édition imprimée

Date de publication : 31 octobre 1993

Pagination : $45-53$

ISBN : 2-8257-0485-7

ISSN : 1662-372X

Référence électronique

Ewa Dahlig, « Monophonie, hétérophonie et poly(?)phonie dans le jeu du violon traditionnel en Pologne », Cahiers d'ethnomusicologie [En ligne], 6 | 1993, mis en ligne le 02 janvier 2012, consulté le 20 avril 2019. URL : http://journals.openedition.org/ethnomusicologie/1386

Ce document a été généré automatiquement le 20 avril 2019.

Tous droits réservés 


\title{
Monophonie, hétérophonie et poly (?)phonie dans le jeu du violon traditionnel en Pologne
}

\author{
Mono-, hetero- (and poly-?)phony in traditional fiddling in Poland
}

\section{Ewa Dahlig}

Traduction : Isabelle Schulte-Tenckhoff

\section{NOTE DE L'ÉDITEUR}

Traduit de l'anglais.

1 Dans la musique traditionnelle polonaise, le violoniste, ou plutôt le violoneux, semble jouer un rôle capital; rares sont en effet les ensembles qui s'en passent. Même un seul instrument d'accompagnement suffit pour constituer, avec le violon, l'ensemble le plus simple.

2 L'accompagnement assume deux fonctions fondamentales. La première, particulièrement importante pour la musique de danse prépondérante dans la musique populaire instrumentale en Pologne, consiste à donner le rythme. L'accompagnement rythmique, joué principalement sur le petit tambour sur cadre tendu d'une seule membrane, assure l'organisation temporelle, c'est-à-dire horizontale, du jeu musical.

3 Le second type d'accompagnement est un bourdon continu ordonnant, non pas le temps mais l'espace sonore. Servant de point de référence à la ligne mélodique, le bourdon assure l'organisation verticale de l'interprétation.

4 Dans la Pologne ancienne, les types d'accompagnement que je viens d'évoquer étaient fort répandus. Une gravure sur bois du XVII ${ }^{\mathrm{e}}$ siècle représentant un couple de danseurs et un ensemble composé d'une cornemuse, d'une vièle, d'une flûte à une main et d'un tambour atteste que l'accompagnement purement rythmique et le bourdon pouvaient coexister. Si cette pratique n'a pas perduré, il n'en existe pas moins actuellement un type 
d'accompagnement combinant les propriétés de l'un et de l'autre, à savoir un bourdon rythmique qui ordonne la structure musicale à la fois verticalement et horizontalement.

A l'exception de certaines localités dans l'ouest et le sud de la Pologne où l'on rencontre des ensembles à bourdon composés d'un violon et d'une cornemuse, le bourdon rythmique est prédominant dans tout le pays. Il semble en exister trois variantes de base, que je désignerais par les qualificatifs suivants: bourdon de timbre, bourdon de hauteur de son, et bourdon harmonique.

En ce qui concerne le bourdon rythmique de timbre, il est exécuté sur un petit tambour à une membrane auquel sont attachées des cymbalettes. Son rôle peut se limiter à une seule mesure (exemple 1), mais il peut aussi introduire des motifs sophistiqués. Le témoignage le plus ancien quant à l'utilisation du tambour comme instrument accompagnant la musique de danse populaire remonte au XVI ${ }^{\mathrm{e}}$ siècle. Mais cette pratique peut fort bien être plus ancienne.

Exemple 1: Bourdon rythmique de timbre (tambour)

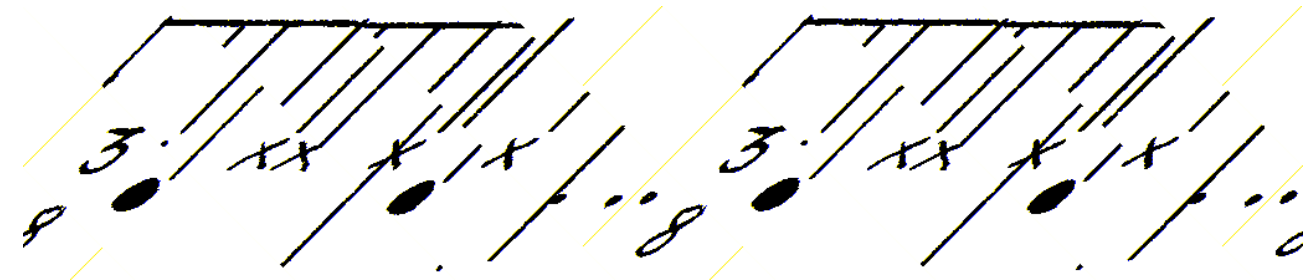

7 Un bourdon rythmique de hauteur de son est un ostinato joué sur les cordes à vide de l'instrument de basse (exemple 2). L'histoire de la partie de basse demeure un mystère. Bien que les sources iconographiques les plus anciennes représentant des contrebasses populaires datent du XVIII ${ }^{\mathrm{e}}$ siècle, il est probable que diverses formes plus rudimentaires aient rempli une fonction similaire dans un passé plus reculé.

Exemple 2: Bourdon rythmique de hauteur de son (contrebasse)

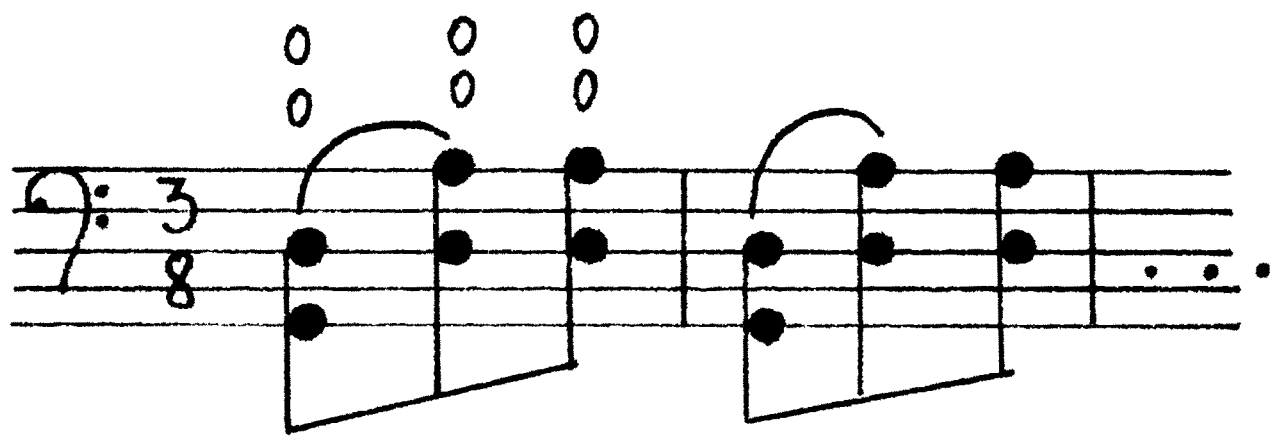

Le bourdon rythmique harmonique est généralement exécuté sur le second violon, et dans le sud-est de la Pologne où l'on pratique le tympanon, il l'est également sur ce dernier. Ce type d'accompagnement appelé sekund est réduit à une formule rythmique en contre-temps jouée en double corde qui correspond du point de vue harmonique à la ligne mélodique (exemple 3). Il utilise le plus souvent des tierces, mais aussi des sixtes, des quintes et des septièmes, exceptionnellement des quartes. D'une manière générale, on constate une tendance à l'ergonomie maximale. Le second violoneux essaie d'éviter tout changement de corde, dans la mesure du possible. Habituellement, il joue les cordes de la et de ré, ou celles de ré et de sol, tout en créant des intervalles suivant le déroulement 
harmonique de la mélodie. Ce type d'accompagnement, rigoureusement lié à l'interprétation en majeur-mineur du répertoire, ne remonte pas plus loin que la seconde moitié du XIX ${ }^{\mathrm{e}}$ siècle. Dans de nombreuses localités, il est d'émergence assez récente. Il convient de mentionner les survivances d'une pratique apparemment plus ancienne: un accompagnement en contre-temps joué en double corde accidentelle.

Exemple 3: Bourdon rythmico-harmonique (contrebasse et violon)

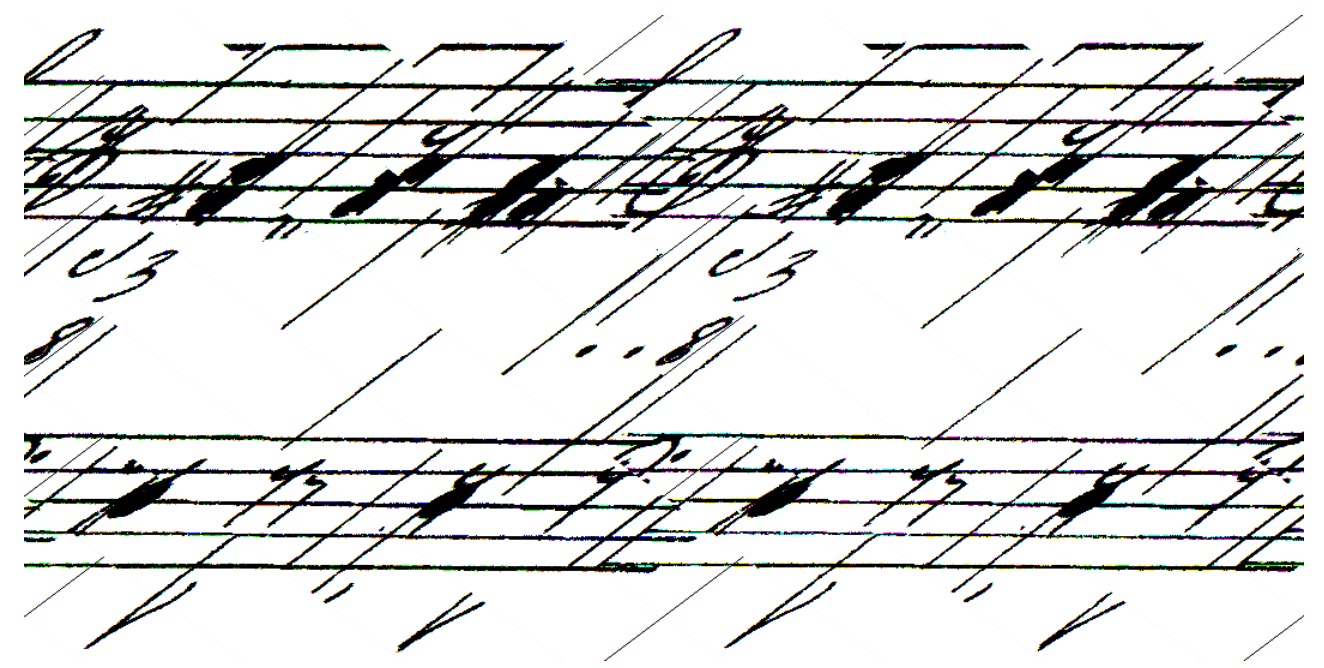

Lorsque le second violon se joint à l'ensemble, le rôle de la contrebasse n'est que de marquer le rythme. Ces deux instruments créent, d'une façon complémentaire, l'ensemble de la structure rythmique et harmonique, si bien que le contrebassiste doit presser les cordes.

Fig. 1: Kazimierz Czubakowski, tambour sur cadre, et Marian Bujak, violon. (Szydłowiec, 1981)

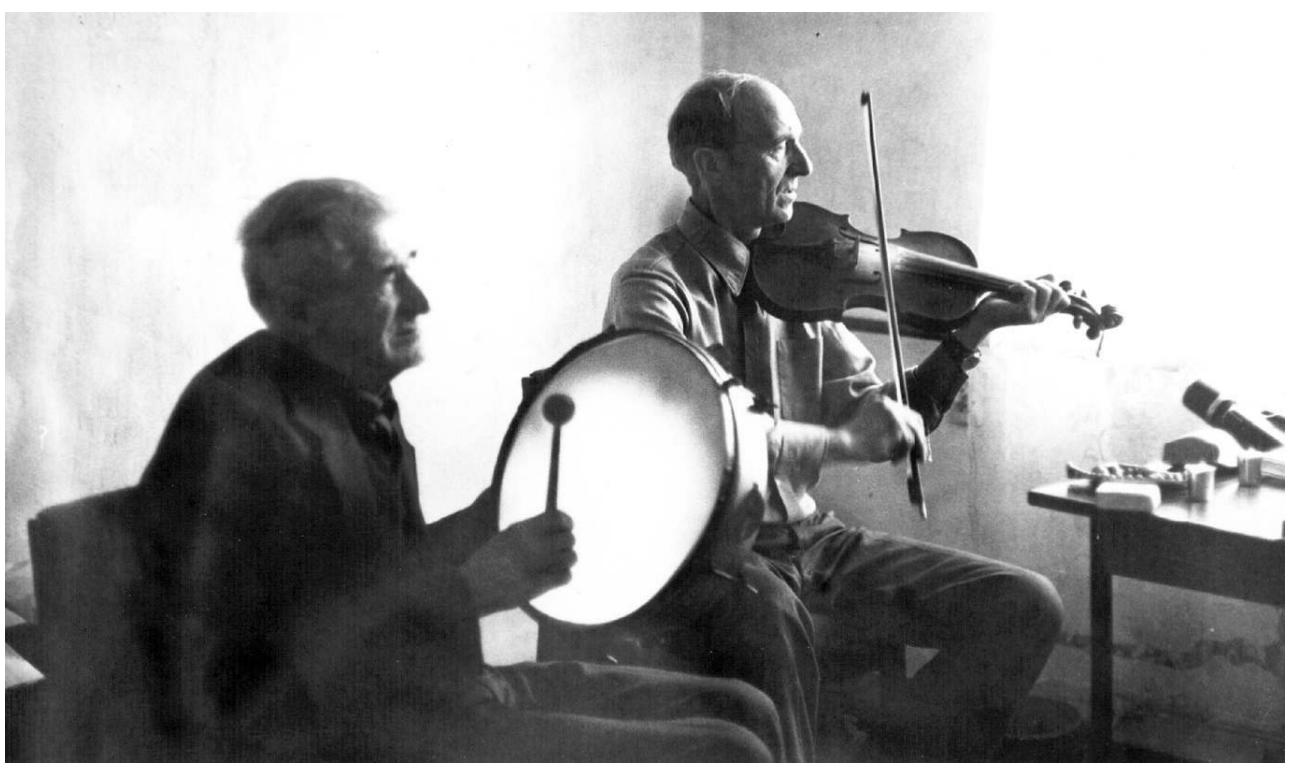

Photo: Ewa Dahlig

10 Mais le second violon peut aussi jouer obligato, ce qui double la mélodie à l'octave inférieure, parfois aussi à un intervalle de tierce ou de sixte. Comme le second violoneux 
est généralement moins habile que le premier, il faut que leurs parties respectives soient clairement différenciées.

11 Considérons maintenant les résultats d'une superposition des parties individuelles (exemple 4). La texture musicale implique deux strates obligatoires, la mélodie et la base, c'est-à-dire un, deux et parfois même trois types de bourdon rythmique. Ce dernier est si nécessaire que - d'après un de nos informateurs - le manque d'instruments d'accompagnement amena un participant lors d'une fête où l'on dansait, à frapper le rythme sur les cordes graves du violon qui ne sont pas utilisées dans le jeu. Deux personnes créaient ainsi les deux strates obligatoires sur le même instrument.

La troisième strate est une ornementation verticale facultative, dont des éléments apparaissent même si l'ensemble ne compte qu'un seul violon; aucun bon joueur ne se limite, en fait, à ne produire que la simple mélodie. Lorsqu'il joue sur l'accompagnement d'une contrebasse ou d'un tambour, le violon doit montrer toute sa créativité et toute sa virtuosité s'il souhaite correspondre à l'idéal esthétique populaire, qui consiste à créer une texture musicale d'une grande densité horizontale aussi bien que verticale. Horizontalement, la mélodie est remplie de mélismes et de formules rythmiques; verticalement, elle l'est de ce qui reste d'un bourdon exécuté sur les cordes à vide. Le jeu en cordes doubles apparaît aussi occasionnellement. D'une manière générale, on constate la règle suivante: moins l'ensemble compte d'instruments, plus la partie du violon est ornée et présente.
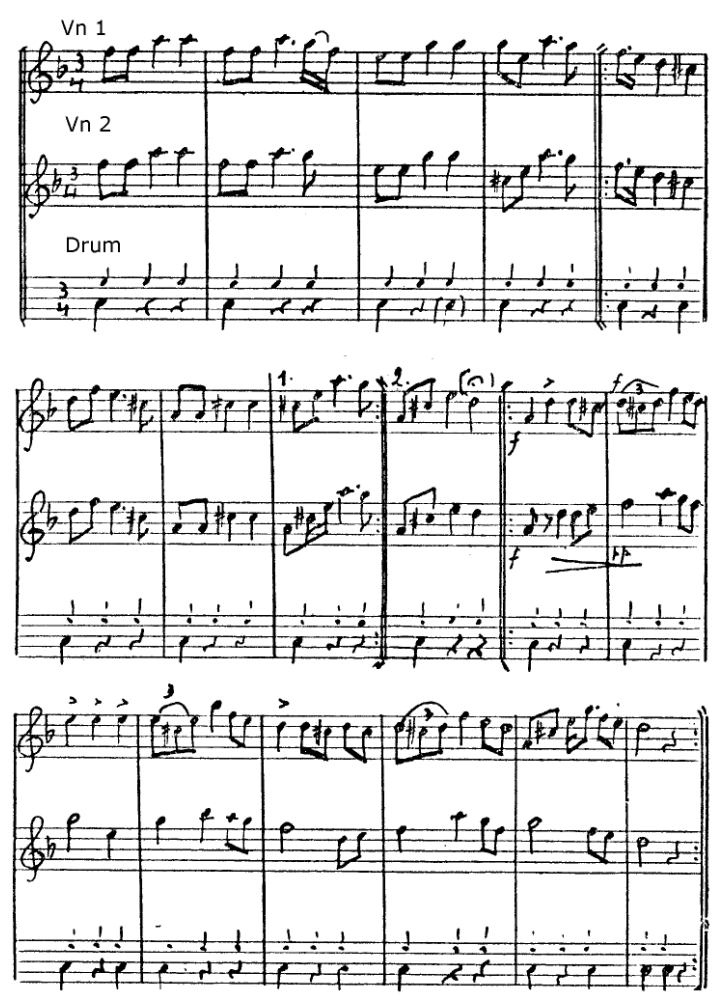


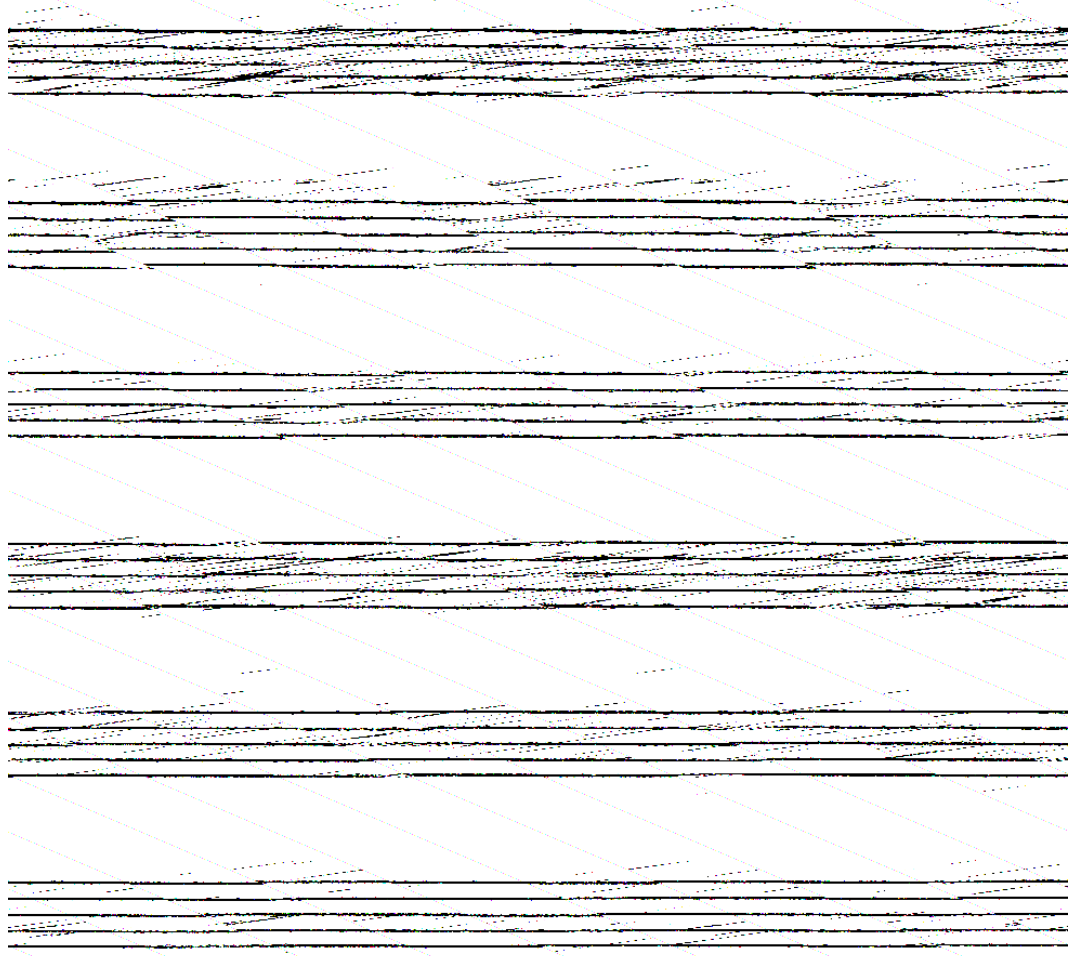

«Teatr Ludowy », 1933, N³, pp. 34-35

13 Dans les ensembles à deux violons, le meilleur (prym) n'a plus besoin d'orner la mélodie verticalement; c'est le second violon qui s'en charge. Selon qu'il joue un sekund non harmonique ou harmonique, voire un obligato, le remplissage vertical peut être de nature quantitative ou qualitative.

14 S'il n'y a que les strates obligatoires, une partie de violon reste monophonique et se déploie sur l'arrière-fond d'un bourdon de timbre ou de hauteur de son. Il en résulte alors l'homophonie. La même chose se passe lorsque la partie appelée sekund se joint à la structure. Si toutefois le second violon introduit l'obligato, on constate l'émergence de rapports hétérophoniques. 
Exemple 4: Stratification de l'espace sonore dans les ensembles à cordes de Pologne

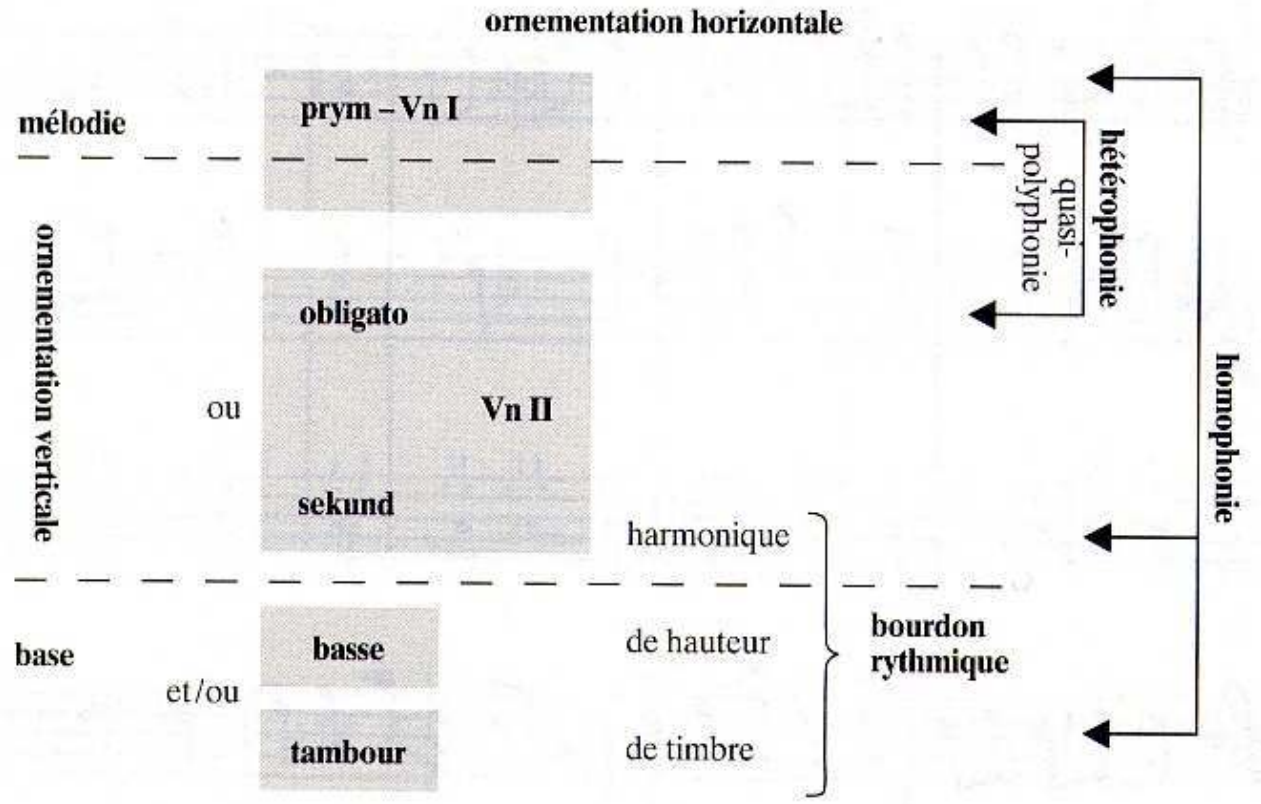

On le voit clairement: dans les ensembles à violon polonais, il n'y a pas de jeu polyphonique au sens strict du terme. De plus, la polyphonie en tant que telle est étrangère à la culture musicale populaire de Pologne. Cependant, dans le répertoire instrumental traditionnel fondé principalement sur des modèles vocaux, il y a quelques symptômes de quasi-polyphonie, c'est-à-dire des tentatives allant dans le sens d'une différenciation partielle des parties mélodiques.

Fig. 2: Wincenty Oleszczak, premier violon, Franciszek Paluch, tambour sur cadre, Tadeusz Oleszczak, sekund. (Bil-goraj, 1982)

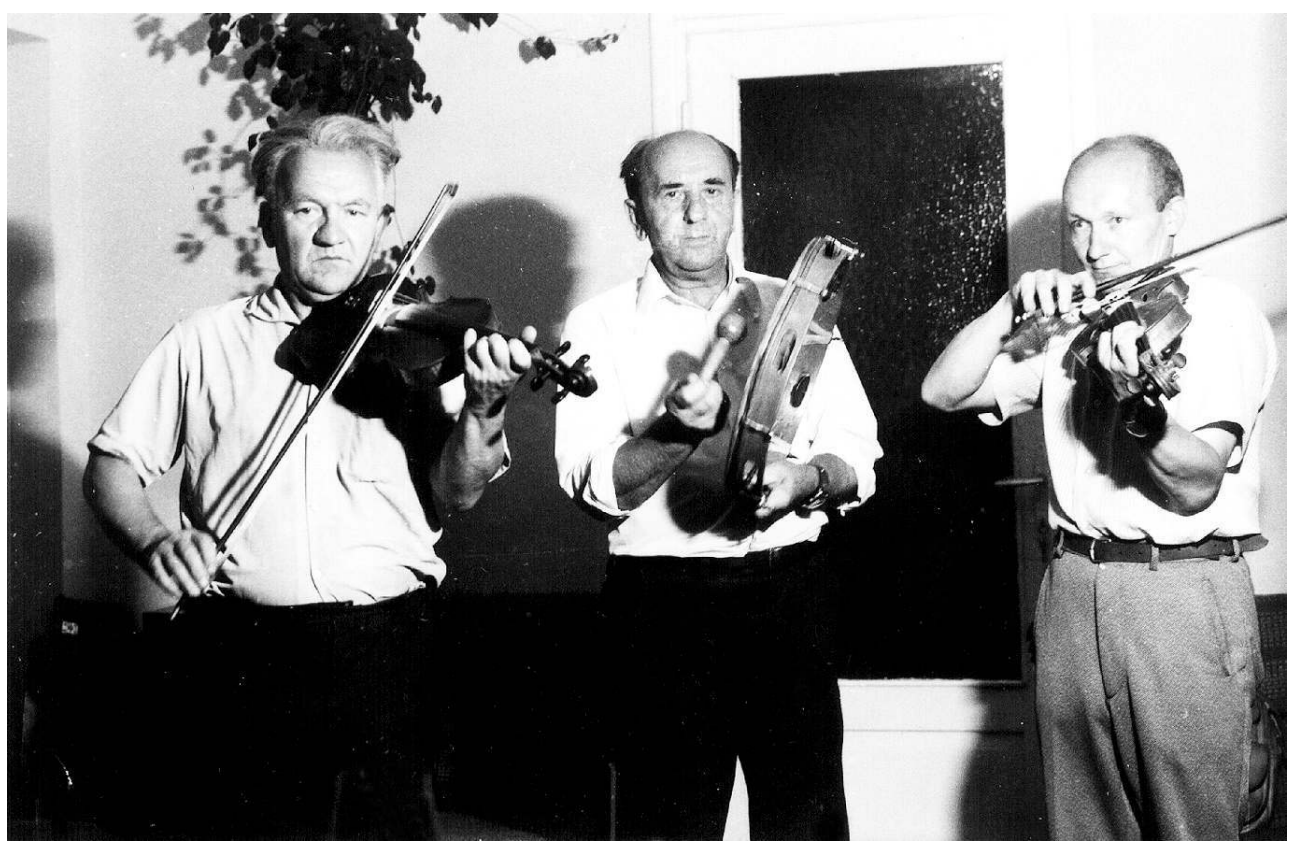

Photo: Ewa Dahlig 
Tout d'abord, même si l'ensemble ne compte qu'un seul violon, on voit que son rôle consiste à transformer le modèle vocal plutôt qu'à le reproduire. Si les versions vocale et instrumentale de la mélodie apparaissent simultanément, on constate que l'instrument joue le rôle d'un riche contrepoint. Or, comme il sont interprétés en alternance (et que parfois la version vocale est omise) il faudrait peut-être parler ici d'une quasi-polyphonie non synchronique. Il faut admettre, bien sûr, que les voix, qui ne résonnent que dans l'esprit des musiciens, soient incluses dans notre concept de polyphonie.

Deuxièmement, lorsque le joueur d'obligato veut rendre sa partie moins schématique, ses tentatives de différencier la ligne mélodique apparaissent comme des éléments d'une quasi-polyphonie.

La quasi-polyphonie, comprise ici comme le niveau le plus élaboré de l'hétérophonie, s'intensifie au fur et à mesure que s'accroît la densité horizontale des deux parties de violon. Les raisons de ce phénomène sont assez complexes, et elles obéissent à divers facteurs (exemple 5).

Certaines conditions préliminaires doivent être remplies pour qu'il puisse y avoir polyphonie. Tout d'abord, pour devenir un bon joueur de prym, le violoneux doit avoir des aptitudes physiques et psychiques. L'étape suivante consiste à suivre un apprentissage, puis à se développer en passant par toute la hiérarchie de la pratique musicale. De nombreux violoneux affirment que, même lorsqu'ils savaient jouer correctement du violon, ils devaient commencer par jouer du tambour ou de la contrebasse. Puis, après quelque temps passé à exécuter la partie de sekund ou d'obligato, ils étaient plus ou moins préparés pour être joueurs de prym. Ainsi la capacité de "polyphoniser», point culminant du développement de la personnalité du joueur de violon, émerge seulement à un moment déterminé de sa vie.

Exemple 5: Le contexte de la quasi-polyphonie dans les ensembles à cordes de Pologne

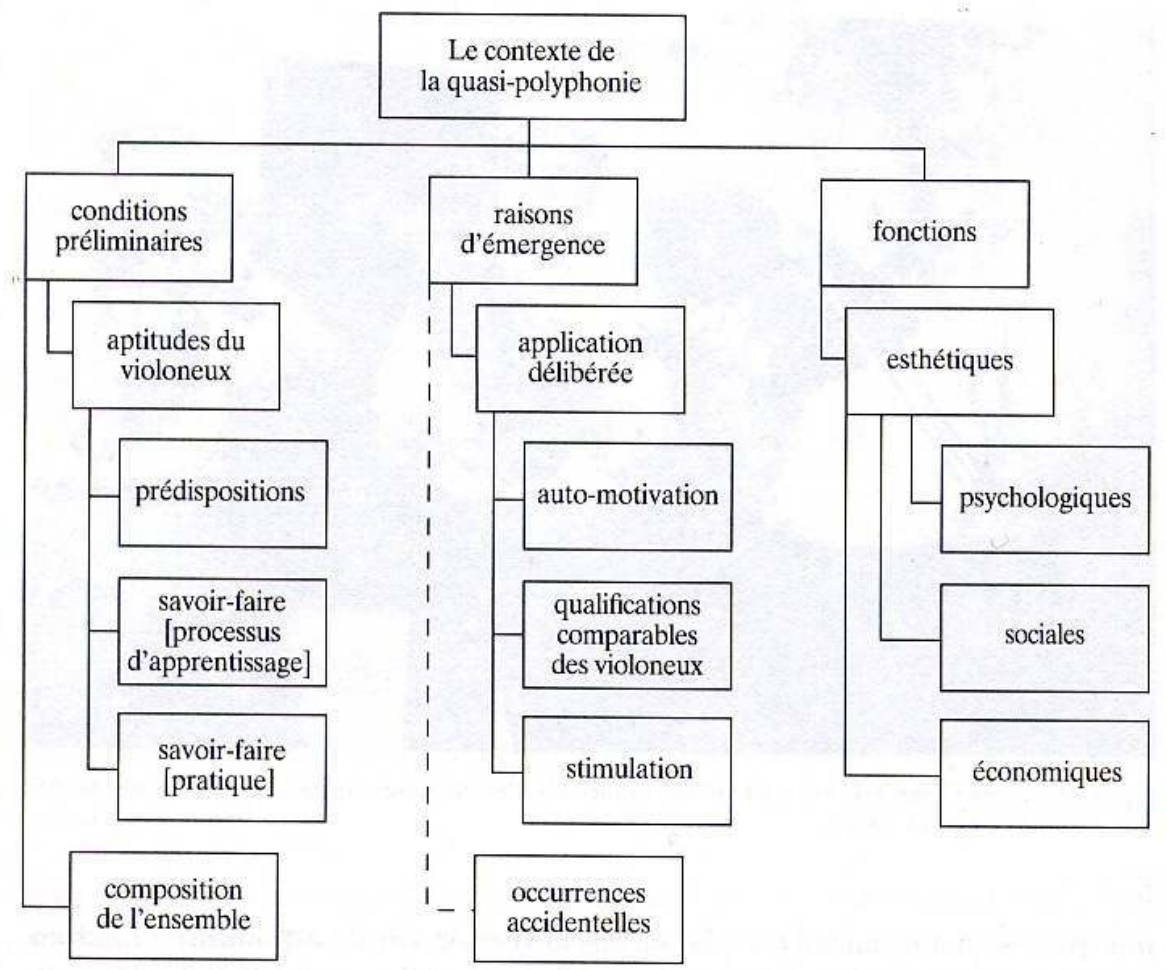


20 Il y a aussi des conditions d'ordre social. Seuls les paysans les plus aisés pouvaient engager de grands ensembles comprenant plus d'une partie mélodique. Ainsi peut-on dire qu'il n'y avait guère de polyphonie pour les pauvres...

21 Il y a deux raisons à la polyphonie (ou à l'hétérophonie, si l'on se place au niveau inférieur). Son application peut répondre aux motivations du joueur qui tente de satisfaire ainsi ses besoins d'expression et de créativité. Un autre facteur est l'existence de qualifications et d'aspirations comparables entre deux violoneux appartenant au même ensemble, mais c'est assez rare, car chaque bon violoneux essaie de former son propre ensemble plutôt que de jouer l'obligato dans un autre. Il y a toutefois des ensembles de familles où deux frères jouent en alternance les parties de prym et de sekund /obligato.

22 Dans les bons ensembles, les musiciens s'inspirent mutuellement et sont motivés par l'événement musical et la danse. Certains, en particulier les violoneux, semblent même tomber dans un état proche de la transe. Un autre facteur de stimulation est la concurrence entre ensembles. L'événement musical et choréographique le plus important dans la vie des paysans était la noce. Juste après la cérémonie, on se rendait à l'auberge la plus proche, où les invités et les ensembles de diverses noces avaient l'habitude de se retrouver. Les musiciens devaient rivaliser, car le meilleur ensemble était le mieux rémunéré.

23 Mentionnons en passant l'hétérophonie accidentelle résultant d'une erreur, d'une confusion, d'un mauvais accord ou même de l'incompétence du joueur d'obligato.

24 Les fonctions de la quasi-polyphonie sont strictement liées aux circonstances de son application, déjà évoquées. La fonction esthétique, c'est-à-dire le fait d'embellir le jeu musical, est ici la plus évidente. Mais elle est complétée par des effets non musicaux d'ordre psychologique, social et aussi économique. La créativité satisfait les besoins affectifs des violoneux, mais elle contribue aussi au prestige qu'ils acquièrent face à la société locale, prestige qui entraîne à son tour un revenu plus élevé.

En résumé, la quasi-polyphonie dans le répertoire des ensemble à cordes en Pologne doit être considérée comme un phénomène de nature non musicale ayant sa propre dynamique. Conditionnée par des facteurs psychologiques et sociologiques, elle s'insère dans le contexte global de la vie paysanne. En ce qui me concerne, la monophonie, l'hétérophonie et la quasi-polyphonie apparaissent, non pas comme trois types distincts, mais plutôt comme trois degrés d'un même processus, c'est-à-dire la plus haute condensation bidimensionnelle de réalisation.

\section{RÉSUMÉS}

The fiddle is the most widespread folk musical instrument in Poland and appears in a variety of ensembles. It is never played in a purely monophonic way, although its possibilities for polyphony are limited. The author describes different forms of co-playwithin fiddle ensembles, such as the ancient practice of emitting a drone, the development of a counterpoint to the 
melodic pattern, or the elaboration ofheterophonic versions set one octave below the melody. She also refers to psychological aspects of fiddling, including the induction of a trance-like state, as well as to the social factors determining thestructure of the ensemble and the musical role of thefiddler.

\section{AUTEURS}

\section{EWA DAHLIG}

Ewa Dahlig a étudié à l'Institut d'ethnomusicologie de l'Université de Varsovie, où elle a obtenu son doctorat en 1990. Depuis 1982, elle travaille à l'Institut d'Art de l'Académie polonaise des sciences. Elle s'intéresse en particulier aux instruments de musique populaire, à la musique instrumentale, ainsi qu'à l'utilisation de l'informatique dans l'analyse musicale. Elle a menée des recherches sur le terrain en Pologne, et elle est l'auteur de Ludowa gra skrzypcowa w Kieleckiem (Le jeu du violon dans la région de Kielce), paru en 1991. 